\title{
Results from a family and DNA based active identification programme for familial hypercholesterolaemia
}

\author{
A H A ten Asbroek, P J Marang-van de Mheen, J C Defesche, J J P Kastelein, \\ L J Gunning-Schepers
}

Heterozygous familial hypercholesterolaemia $(\mathrm{FH})$ is a common inborn error of lipoprotein metabolism, which strongly predisposes for coronary artery disease and premature cardiac death. ${ }^{1}$ In 1994, a family based active identification programme for $\mathrm{FH}$ was implemented in the Netherlands. ${ }^{2}$ It is based on DNA diagnosis of the LDL-receptor gene mutation, which enables us to search selectively for patients in a high risk population. The programme initially targets first and second degree relatives of $\mathrm{FH}$ probands (diagnosed at Lipid Research Clinics throughout the country) and extends further into the family only when new patients are identified. The programme aims to identify mutation carriers and to refer them to Lipid Research Clinics for extensive individual risk assessment and, if necessary, treatment. As no carefully collected data are available for cholesterol levels among the general population of LDL-receptor gene mutation carriers, the large majority of whom are asymptomatic, we studied the prevalence of hypercholesterolaemia among screenees with a proved LDLreceptor gene mutation.

\section{Methods and Results}

Between 1994 and 19982814 adults were screened. The estimated response rate was constant over the years at $90 \% .^{3}{ }^{4}$ For reasons of comparison with available population data for total serum cholesterol levels, ${ }^{5}$ we selected those who were between 20 and 60 years of age (1856 screenees). Depending on the available funds in the screening programme, which were lacking in certain periods, single cholesterol measurements were taken at the time of screening. Therefore, we analysed the data of all 1005 persons who had DNA test results as well as cholesterol measurements. These were a non-selective sample of the 1856 screenees between 20 and 60 years of age. Cholesterol was measured using commercially available kits (Boehringer Mannheim, Mannheim, Germany). Genomic DNA was isolated from the leucocyte fraction of $10 \mathrm{ml}$ of freshly collected blood, followed by polymerase chain reaction and restriction enzyme analysis.
From the perspective of the screening programme, the screenees that are already treated cannot be considered as new cases and they do not benefit from the screening programme in the same manner as newly identified cases. Therefore, we present the prevalence of hypercholesterolaemia among all screenees as well as the prevalence of hypercholesterolaemia among those not yet treated with HMG-CoA reductase inhibitors.

Hypercholesterolaemia was defined as either an untreated total cholesterol (TC) level above the 95th centile for age and sex in the Dutch population (table 1$),{ }^{5}$ or as receiving HMG Co-A reductase inhibitors. We also show the total cholesterol distribution for the untreated screenees using conventional cut off points $(<6.5,6.5-7.9, \geqslant 8 \mathrm{mmol} / \mathrm{l})$. All LDL-receptor gene mutation carriers were heterozygotes. None of the screenees had been tested for a LDL-receptor gene mutation before.

Table 2 shows the results for the screened population.

It is evident that each age category contains LDL-receptor gene mutation carriers who do not have hypercholesterolaemia: $19.8 \%$ in all men, $32.3 \%$ in untreated men and $16.7 \%$ in women, $28.7 \%$ in untreated women. Furthermore, it is shown that the prevalence of mutation carriers among all screenees tends to be lower in the older age groups. This is probably the result of selective mortality. However, the prevalence of mutation carriers among untreated screenees is also lower in the older age groups. This is not purely the result of selective mortality but it is mainly attributable to the fact that an increasing proportion of those screened in the older age groups is already treated with cholesterol lowering drugs, and more in mutation carriers than in those without a mutation as they have generally higher cholesterol levels. This might also explain why the prevalence of hypercholesterolaemia in untreated female mutation carriers is lower in the older age groups.

Table 1 MORGEN Project data 1996-1997: mean (SD) total serum cholesterol (TC) as well as 95th centile (C95) in the general Dutch population by sex and age group

\begin{tabular}{|c|c|c|c|c|c|c|c|c|}
\hline \multirow[b]{2}{*}{ Age group (y) } & \multicolumn{4}{|l|}{ Men } & \multicolumn{4}{|l|}{ Women } \\
\hline & $20-29$ & $30-39$ & $40-49$ & $50-59$ & $20-29$ & $30-39$ & $40-49$ & $50-59$ \\
\hline Number & 589 & 890 & 1210 & 1030 & 843 & 1140 & 1450 & 1139 \\
\hline Mean TC (SD) & $4.4(0.9)$ & $5.0(1.0)$ & $5.5(1.0)$ & $5.5(1.0)$ & $4.6(0.8)$ & $4.8(0.8)$ & $5.1(0.9)$ & $5.7(1.0)$ \\
\hline C95 & 5.8 & 6.7 & 7.2 & 7.1 & 6.1 & 6.3 & 6.7 & 7.4 \\
\hline
\end{tabular}




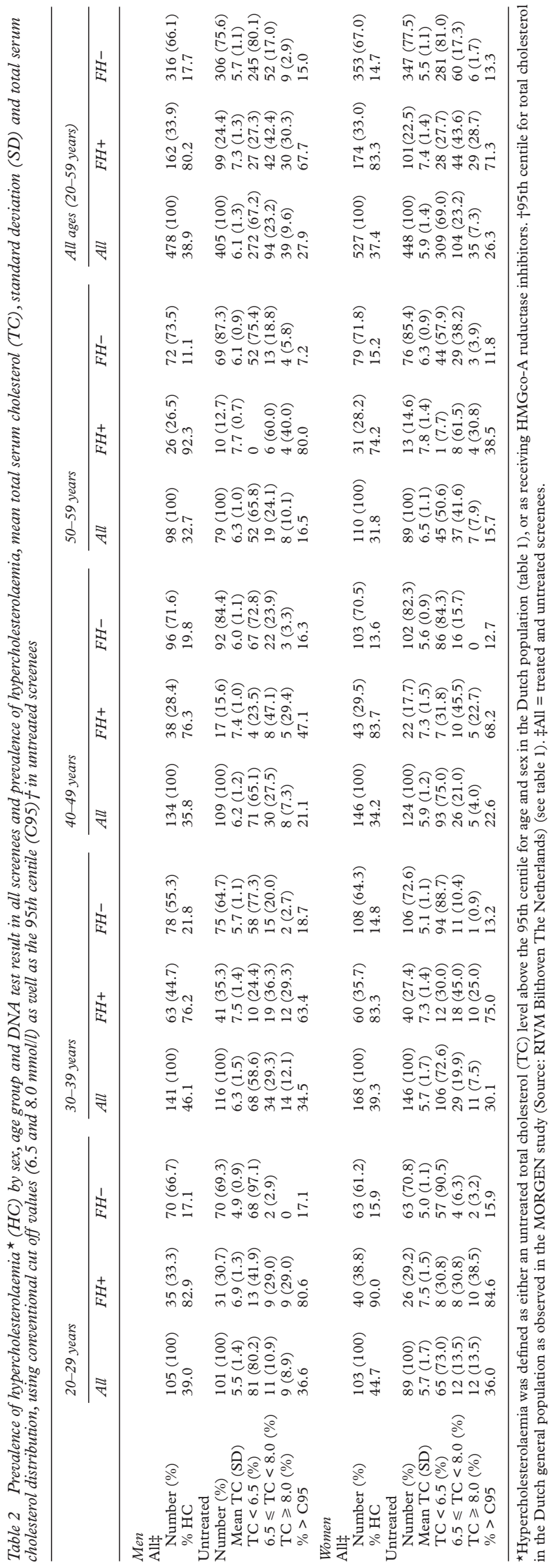

\section{Discussion}

These data have not been shown before in such a large and well defined cohort, and have important consequences for case finding strategies for inherited disorders of lipoprotein metabolism with a known molecular basis. Our study shows that if in a high risk population of yet untreated, mainly asymptomatic mutation carriers, a single TC level would be used for the diagnosis of $\mathrm{FH}$ rather than the current gold standard-that is, the presence of a LDLreceptor gene mutation - the diagnosis would be missed in more than a quarter of the $\mathrm{FH}$ patients.

As is shown by others, FH is not fully penetrant from birth onwards. ${ }^{6}$ It has not been reported before, however, to which extent the genetic disorder causes hypercholesterolaemia in a population of mainly asymptomatic adult relatives of genetically diagnosed patients as is shown by this study.

The high prevalence of "lower" TC levels in our cohort may well reflect a combination of factors like patients' adherence to low calorie diets for weight loss, intercurrent illness of infectious nature, a better general health of participants in a screening programme, or a protective genetic constitution. In addition, because cholesterol levels vary with LDLreceptor mutation, ${ }^{7}$ we cannot exclude that our findings of normal cholesterol levels in LDLreceptor gene mutation carriers are the result of screening for mutations that may result in a milder than expected phenotype as reported elsewhere, albeit very unlikely. Nevertheless, even though a single measurement of cholesterol is not very reliable when assessing the individual cardiovascular disease risk, for the purpose of assessing the cholesterol values on a population level a single measurement can be used. $^{8}$

The prevalence of hypercholesterolaemia in those without a LDL-receptor gene mutation is higher than in the general population, which indicates that in this high risk population probably also other factors than the LDL-receptor gene mutation contribute to the prevalence of hypercholesterolaemia.

The importance of our findings depends largely on whether patients with a LDLreceptor gene mutation but without hypercholesterolaemia, experience an increased risk of coronary heart disease and whether they need the same rigorous treatment as other $\mathrm{FH}$ patients. This is currently unknown. As damage to the vascular wall in FH patients is probably the result of the number of cholesterol years, ${ }^{9}$ a single TC level below the 95 th centile could be falsely reassuring. Comprehensive appraisal of cardiovascular disease risk and cholesterol screening at regular intervals is advised. In a follow up study, we plan to further explore explanations for our findings and to assess whether the risk of coronary heart disease is increased in this group of mutation carriers in whom $\mathrm{FH}$ does not seem to be fully penetrant.

A H A ten Asbroek was the main author and carried out the analyses and interpreted data. P J Marang-van de Mheen 
formulated the research questions, assisted in writing, analyses and interpreting results. J C Defesche participated in data screening and cleaning, assisted in the analyses as well as in the interpretation of the data and assisted in writing the report. J J P Kastelein assisted in writing and interpreting results. L J Gunning-Schepers was principal investigator, formulated the research questions, assisted in writing the report and is head of the study group.

Funding: this study is funded by the Health Research and Development Council (grant number 28-2751).

Conflicts of interest: none.

1 Anonymous. Risk of fatal coronary heart disease in familial hypercholesterolaemia. Scientific Steering Committee on hypercholesterolaemia. Scientific Steering Committee on 1991;303:893-6.

2 Umans-Eckenhausen MA, Defesche JC, Scheerder RL, et al. [Tracing of patients with familial hypercholesterolemia in the Netherlands]. [In Dutch]. Ned Tijdschr Geneeskd 1999;143:1157-61.
3 Stichting Opsporing Erfelijke Hypercholesterolemie StOEH [Foundation for Tracing Hereditary Hypercholesterolemia]. Annual report 1997. Amsterdam: StOEH, 1998.

4 Stichting Opsporing Erfelijke Hypercholesterolemie $\mathrm{S} t \mathrm{OEH}$ Foundation for Tracing Hereditary Hypercholesterolemia]. Annual report 1998. Amsterdam: StOEH, 1999.

5 Blokstra A. [The Project Monitoring Risk Factors and Health, The Netherlands] (MORGEN-project). Annual report 1996. Report no 263200 006. Bilthoven: Rijksinstituut voor Volksgezondheid en Milieu, 1997.

6 Leonard JV, Whitelaw AG, Wolff $\mathrm{OH}$, et al. Diagnosing familial hypercholesterolaemia in childhood by measuring serum cholesterol. BM7 1977;1:1566-8.

7 Humphries SE, Galton D, Nicholls P. Genetic testing for familial hypercholesterolaemia: practical and ethical issues. QfM 1997;90:169-81

8 Verschuren WMM. Blood cholesterol: a public health perspective. Wageningen: Ponsen and Looijen, 1995.

9 Hoeg JM, Feuerstein IM, Tucker EE. Detection and quantitation of calcific atherosclerosis by ultrafast computed tomography in children and young adults with homozygous familial hypercholesterolemia. Arterioscler Thromb 1994;14: 1066-74.

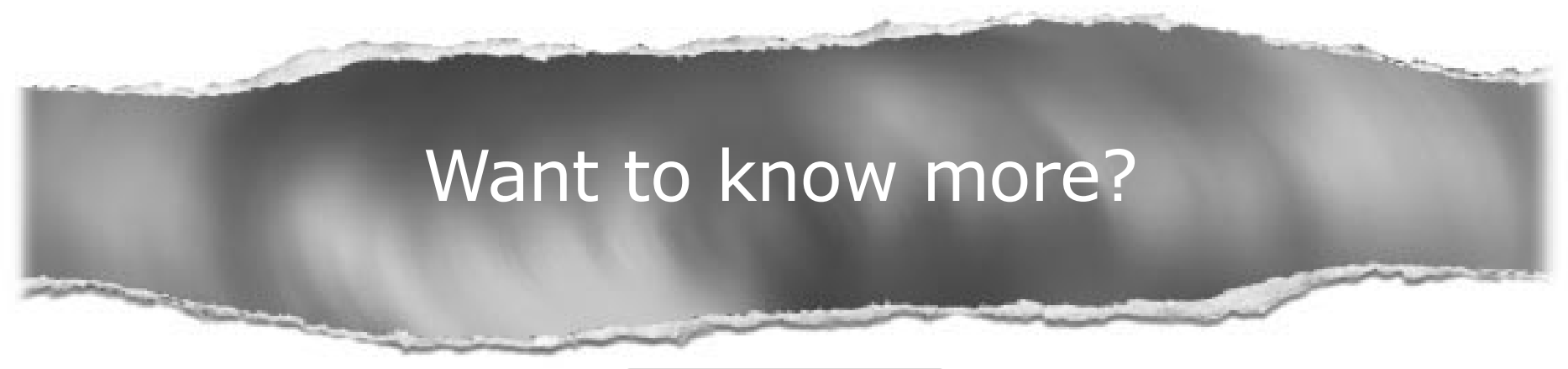

Data supplements

Limited space in printed journals means that interesting data and other material are often edited out of articles; however, limitless cyberspace means that we can include this information online. Look out for additional tables, references, illustrations.

www.jech.com 